\title{
The Volumetric Erosion of Electrical Contacts
}

\author{
John W. McBride
}

\begin{abstract}
In this paper a method for measuring the volume of eroded electrical contacts is presented. The main emphasis is on the measurement of volume relative to a contact support surface, allowing severely eroded contacts to be measured. A noncontact system is described, which allows contacts to be evaluated for volume change without the contact being removed from contact supports. The accuracy of the measurement method is discussed and results show how the volume measurement can be used to evaluate contact performance. Results are presented on the erosion characteristics of $\mathrm{Ag} / \mathrm{SnO}_{2}$ contacts used in automotive relays. A new measurement methodology is presented which will allow for the evaluation of the performance of relay contacts in devices.
\end{abstract}

Index Terms-Automotive relays, contact erosion, noncontact surface measurement.

\section{INTRODUCTION}

$\mathbf{T}$ HE evaluation of the arc erosion of electrical contacts is fundamental to understanding the performance of contact materials and switching devices. The most common measurement of erosion is the mass change of the contacts. In a previous paper, [1], contact erosion was considered in the context of volumetric erosion. It was shown that there were cases where the mass erosion was not sufficient to define the surface changes on a contact. In [1] measurements were made on contact surfaces that were nominally spherical. In [2] an interferometer has been used to measure the surface changes of contactor contacts. The contacts were again nominally spherical.

Fig. 1 shows a range of typical electrical switching contact geomtries, for devices used in low loltage systems:

A) nominally flat surface;

B) nominally spherical surface contact used in low power switching applications;

C) contact with a near vertical side and a spherical contact region.

Type C) is commonly used in automotive relay devices and an analysis of the curved surface commonly shows a nonspherical area where the contact shape has been deformed by a welding process.

Recent trends in low voltage relay devices used in the automotive sector have been toward improving the performance by the optimization of materials. An increase in the voltage supply level from the norm of $12 \mathrm{~V}$ to above $40 \mathrm{~V}$ will require the investigation of new contact arrangements. There is a need to develop

Manuscript received October 6, 1999; revised November 11, 1999. This paper was recommended for publication by Editor B. Jen-Jemaa upon evaluation of the reviewers' comments. This work was presented at the 45th IEEE Holm Conference on Electrical Contacts, Pittsburgh, PA, October 4-6, 1999. This work was supported in part by Chugai.

The author is with the School of Engineering Science, Electro-Mechanical Research Group, University of Southampton, Southampton S017 1BJ, U.K.

Publisher Item Identifier S 1521-3331(00)01919-X.

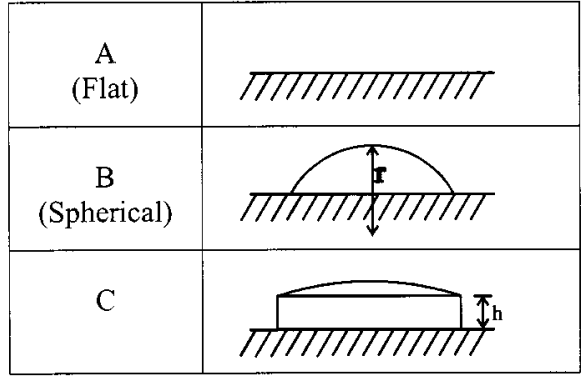

Fig. 1. Typical low voltage electrical contact shapes.

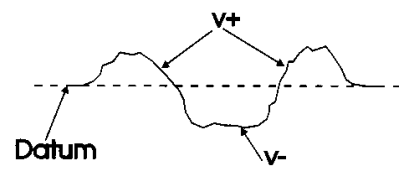

Fig. 2. Two-dimensional erosion profile of an electrical contact surface.

TABLE I

COMPARISON OF THE 3-D SYSTEMS TO

MEASURE THE SURFACES DEFINED IN FIG. 1. (I.F = INTERFEROMETRY)

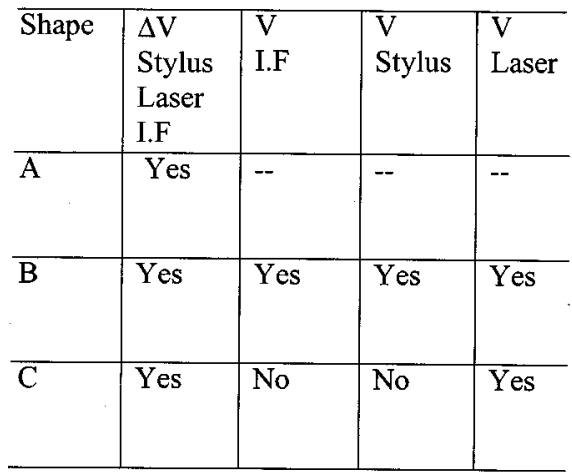

new methodologies for the investigation of contact materials in device studies.

\section{EVALUATION OF ARC EROSION}

In many applications the change of contact mass is used as a parameter to define contact erosion. However there are a number of applications where a mass measurement is not ideal and where volumetric analysis has advantages.

1) Contacts with a very low level of mass change, after a few operations.

2) Contacts where the mass change does not reflect the surface change, [1]. 


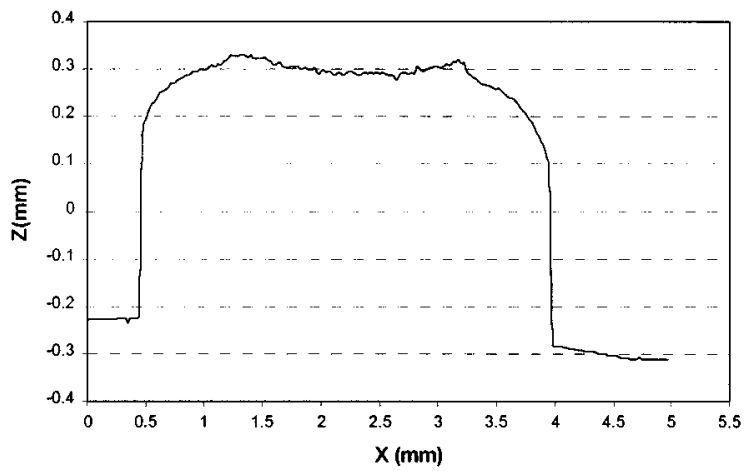

Fig. 3. Two-dimensional scan of eroded contact, using noncontact laser probe.

TABLE II

Automotive Relay Contact SAMPLES TESTEd In A COMMERCIAL RELAY

\begin{tabular}{l|l|l}
\hline $\begin{array}{l}\text { Anode } \\
\text { Contact }\end{array}$ & $\begin{array}{l}\text { Cathode } \\
\text { Contact }\end{array}$ & $\begin{array}{l}\text { Number of } \\
\text { test cycles. }\end{array}$ \\
\hline $1 \mathrm{~A}$ & $1 \mathrm{C}$ & 500 \\
\hline $2 \mathrm{~A}$ & $2 \mathrm{C}$ & 5000 \\
\hline $3 \mathrm{~A}$ & $3 \mathrm{C}$ & 20,000 \\
\hline $4 \mathrm{~A}$ & $4 \mathrm{C}$ & 300,000 \\
\hline
\end{tabular}

3) Measurements where the contacts cannot be removed from a device for mass measurement.

The importance of the volumetric study of contact surfaces was emphasised in [1], and it was shown that cases could occur where there is no net mass change of a surface, although the contacts could show an eroded surface, as shown in Fig. 2. Where the net mass change is zero but the contact surface exhibits a surface change. It was also shown that if the density of the eroded contact material is assumed constant then the net volume change could be used to calculate a mass change for the surface. In the figure the volume change would be calculated relative to the datum surface. Two volumetric measurements can be made.

1) The total volume of the contact above a datum surface, usually the contact support, $(V)$.

2) The change in volume $(\Delta V)$ relative to a datum surface fitted to the contact surface. The change in volume can be defined with reference to Fig. 2:

$$
\Delta V=V_{+}-V_{-} .
$$

In recent studies [1], [2], the volume change $\Delta V$ has been used to measure surface changes on contact type, B. In device studies and in the case where no datum surface can be defined, for example on severely eroded contacts, the volume change measurement is not possible, therefore the total volume measurement method should be used.

\section{SURface ProfiLe Systems}

In the previous paper, [1], methods were reviewed for the measurement of contact surfaces. It was identified that the stylus method offered the standard technique against which the others could be compared. This method has an advantage on electrical contact surfaces where the stylus interaction with the surface can remove loose debris, enabling a picture of the true surface form or shape. In this paper a new system is presented based on laser measurements, and the results used in the previous paper are used for comparison.

Interferometry can be used to measure the erosion of nominally spherical contacts [2]. The interferometer measurement is characterized by the rapid measurement time and the high resolution of data in the $X, Y$ plane. The technique is therefore ideal for rapid measurements, but is limited in the true measurement of the surface, as performed by a stylus. Interferometers functions by making a comparison with a high precision reference surface. The contacts of type $\mathrm{C}$, generally do not show a high degree of surface precision and can be eroded to the extent that the curved surface is removed.

Table I, shows how the various measurement systems can be used in the evaluation of the contact shapes defined in Fig. 1. There are two basic measurement parameters identified, $\Delta V$, for the change in volume from a datum surface and $V$ the volume above the contact base. With a flat contact surface (type A) all three systems will allow for the evaluation of $\Delta V$. The volume measurement relative to a fixed datum is therefore redundant. In the case of a spherical surface (type B) all three systems will allow for both $\Delta V$ and $V$ measurements. In the case of type $\mathrm{C}$ contacts the interferometer and stylus can make a measurement of $\Delta V$ only if the top surface is spherical. The two systems are not able to measure the volume relative to a datum, although in the case of the stylus a method is presented here, but is shown to be inaccurate. Only the laser system is able to measure the volume of type $\mathrm{C}$. The stylus probe is unable to measure the near vertical sides of contact $\mathrm{C}$ since the stylus return motion will damage the measurement system. The laser system although limited in gauge range is a noncontact system, and can therefore measure all of the surfaces in Fig. 1 and obtain measurement of volume changes of electrical contacts relative to the contact base, as identified in Table I.

\section{A. Stylus Based Systems}

A typical stylus based measurement system uses a high precision diamond tip of sub micron radius, which is drawn across a surface. Such devices are able to resolve to $10 \mathrm{~nm}$ in the vertical $Z$ axis making use of an interferometer to measure the $Z$ axis movement. The system used here is the Rank Taylor Hobson Form Talsurf (TALYSURF).

1) Auto-Lift Off Stylus Method: A new stylus based measurement method is presented here which overcomes the requirement to return the stylus over the surface. This allows measurements to be taken of sample type $C$. The new method allows the stylus to be drawn across the contact surfaces in a two dimensions scan of the surface. For the return motion the stylus must lift off the contact surface and a control system is used to position the stylus back on the surface for the next scan. This prevents the stylus being pushed back across the surface to the start position, and thus prevents damage to the measurement system. Each new start position will be subject to a high degree of error in the vertical $Z$ axis. This error is removed in software processing to align all of the start positions to the same value in the $Z$ axis. 


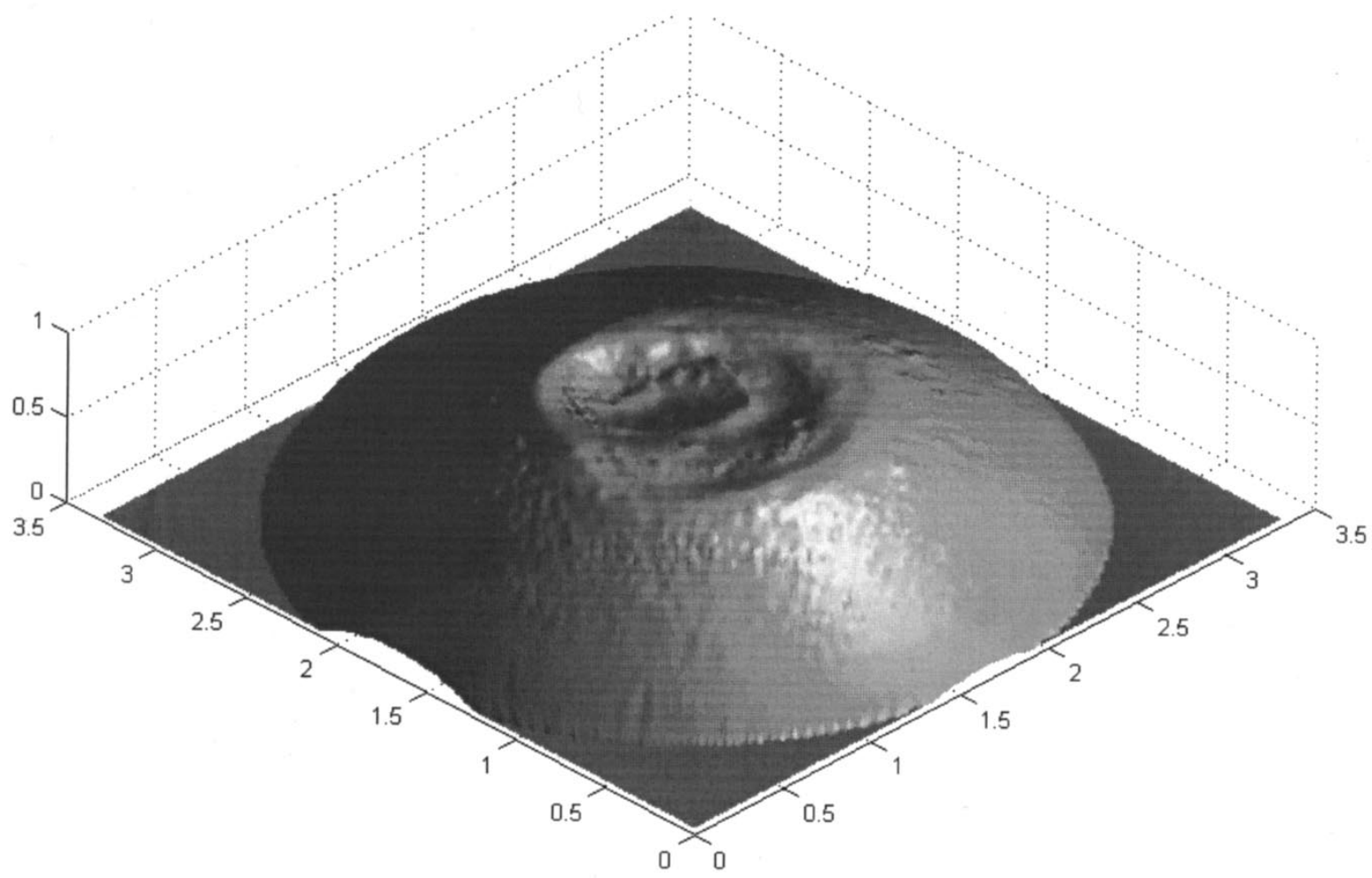

Fig. 4. Anode surface of a spherical $\mathrm{AgCdO}$ contact, after 4000 switching operations at $14.7 \mathrm{~A}$ in a $240 \mathrm{~V}$ ac supply.

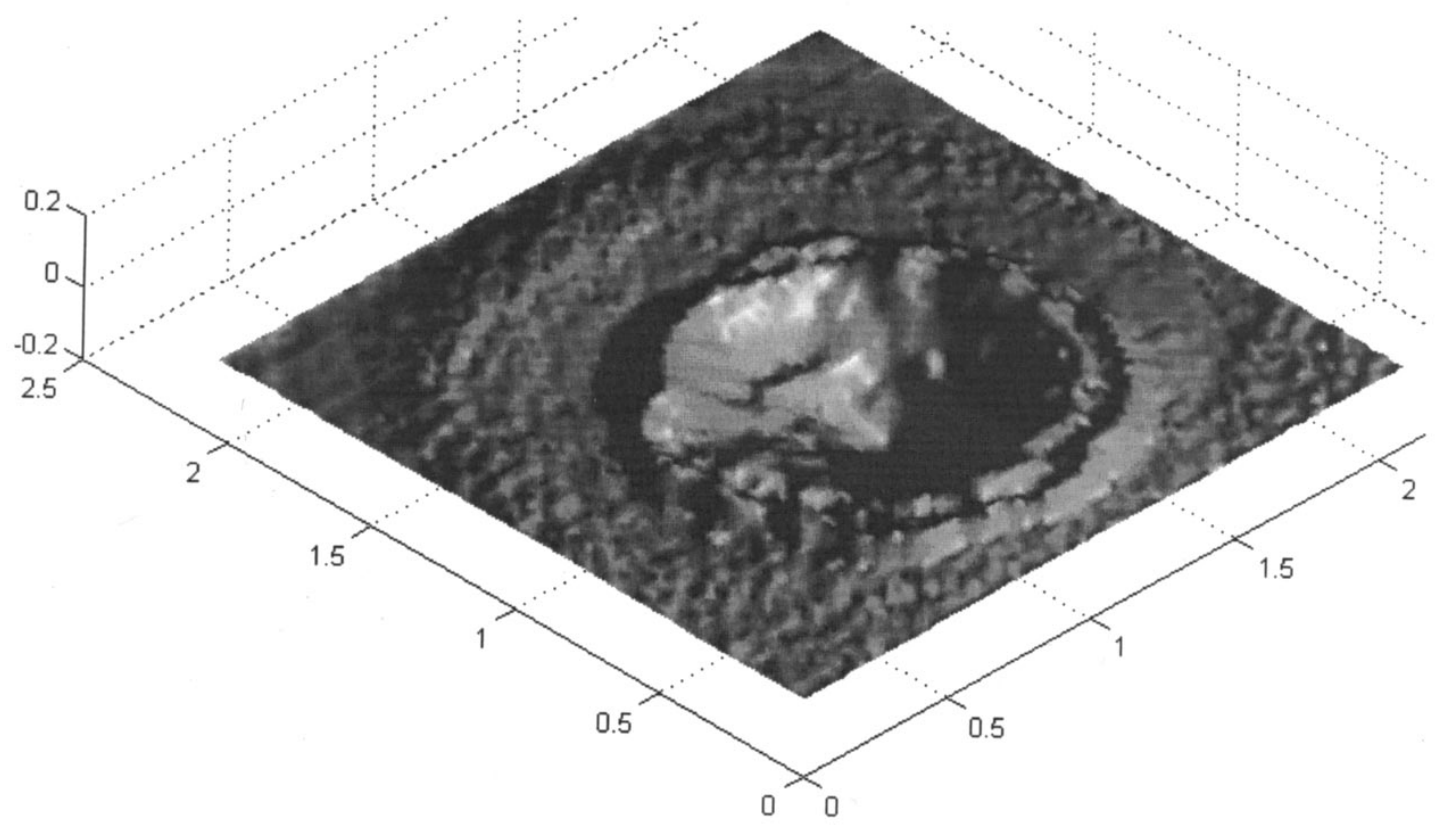

Fig. 5. Stylus probe measurement of zero mass change spherical contact with $2.4 \mathrm{~mm}$ radius removed.

The new method will allow the near vertical sides of the contact shape $\mathrm{C}$ to be measured. Each two-dimensional (2-D) measurement will be subject to a systematic error caused by the stylus flanking error as the sides of the stylus, rather than the stylus tip, ride up the near vertical sides. This will result in volume values that are greater than the true values. 


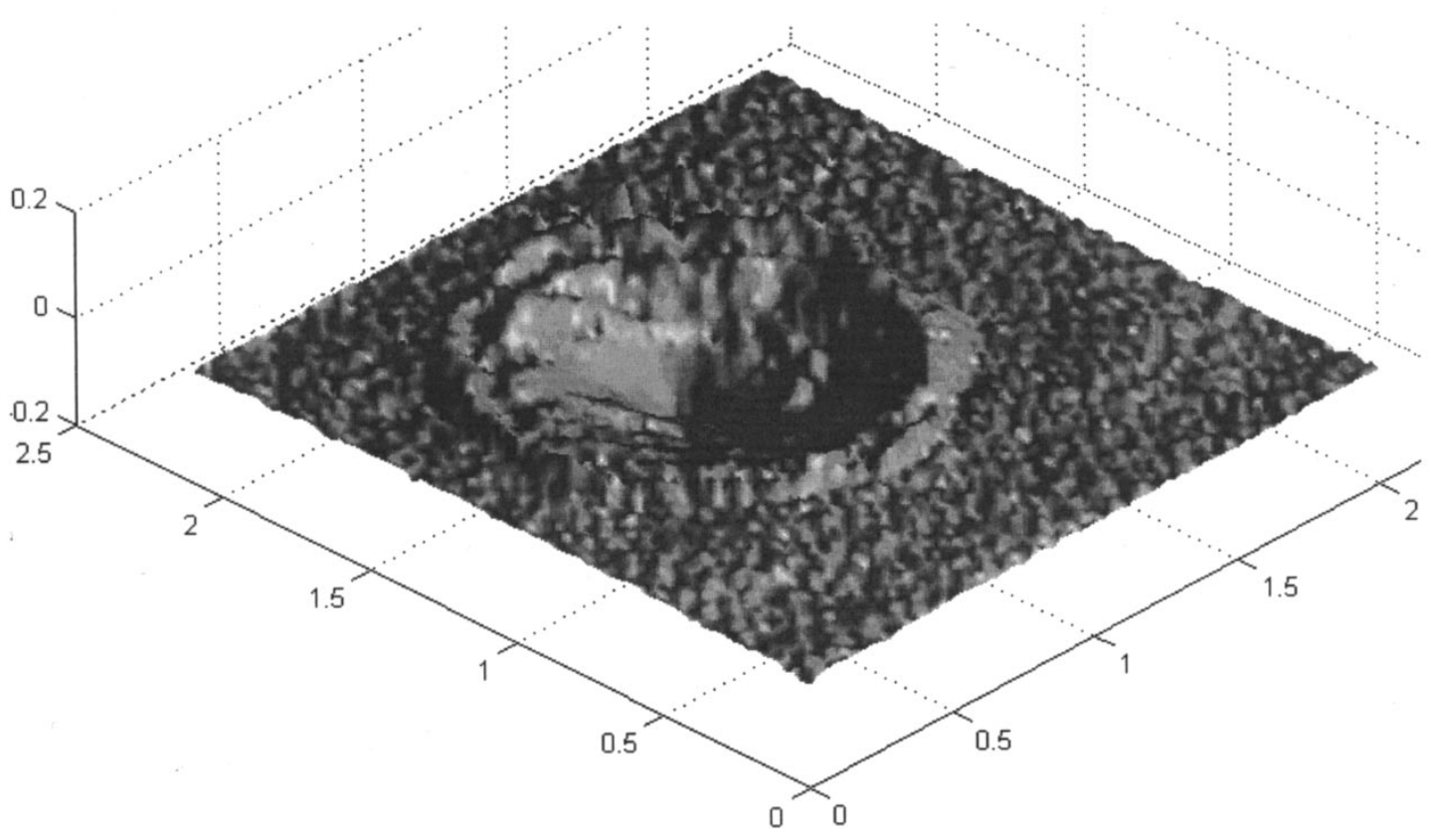

Fig. 6. Laser measurement of zero mass change contact, as shown in Fig. 5

\section{B. Laser Based Instruments}

There are a number of laser based instruments, for the measurement of surfaces. For high precision measurement, as required in the study of contact surfaces, laser triangulation devices do not have sufficient resolution. In the system used in this paper a commercial auto-focus probe has been coupled with high precision $X, Y, Z$ stages. The system is coupled to a software analysis package which allows for full analysis of contact surfaces, [3]-[7]. The main characteristics of the system are as follows; a $Z$ resolution of $0.1 \mu \mathrm{m}$, and the ability to measure near vertical surfaces. This ability is demonstrated in Fig. 3, where a 2-D scan is shown across an eroded contact surface.

The principle limitation is the slow measurement time. The laser must be stationary to make the high precision measurements. The measurement time is therefore limited by the control system.

\section{Measurement Time}

Stylus based systems function such that data is collected continuously as the stylus is drawn across a surface. The time taken for a typical scan of $5 \mathrm{~mm}$ is approximately $30 \mathrm{~s}$. To collect 164 scans will typically take less than $2 \mathrm{~h}$.

The con-focal laser based system described requires that the laser is stationary to make the high precision measurement. The limitation of the laser-based system is therefore the control of the $X$ and $Y$ axis. The present state of the art system requires approximately $5 \mathrm{~min}$ for a single scan, of 100 data points. This leads to a time of approximately $9 \mathrm{~h}$ for 100 scans. For the laser to run on a continuous basis would require significant improvements in con-focal laser measurement systems.

\section{SURFACE ANALYSIS}

A number of numerical methods for volume analysis where compared in a previous paper [1]. It was concluded that best results were achieved using the three-dimensional (3-D) Simpson rule method developed in that paper. In this paper the same numerical scheme will be used in the evaluation of 3-D data sets.

\section{A. Error Analysis}

There are three potential sources of error in the volumetric analysis of surfaces, the error associated with a specific data point in $X, Y, Z$, the error associated with the numerical method used, and the error resulting from the size of the data set.

For the $X, Y, Z$ error, the probe should have the highest resolution possible in $(Z)$ and the tables controlling the movement of the probe or surface $(X, Y)$ should meet the same requirement.

The accuracy of the numerical method has been considered elsewhere. For the evaluation of contact volume changes, the size of the data array used to measure the surface must be as large as possible. In the case of the stylus system the array is set at $164 \times 164$ data points, while in the case of the laser the array size is set at $100 \times 100$. The principle error in the volumetric measurement will be associated with the size of the data set. The larger the data set the smaller the systematic error in the volume calculation. For the same size data set and the same contact geometry, the systematic error in the volumetric measurement will be constant.

\section{B. Spherical Surfaces}

In the previous paper a sphere fitting routine was used to evaluate the best fit surface to eroded contacts of shape B. The 


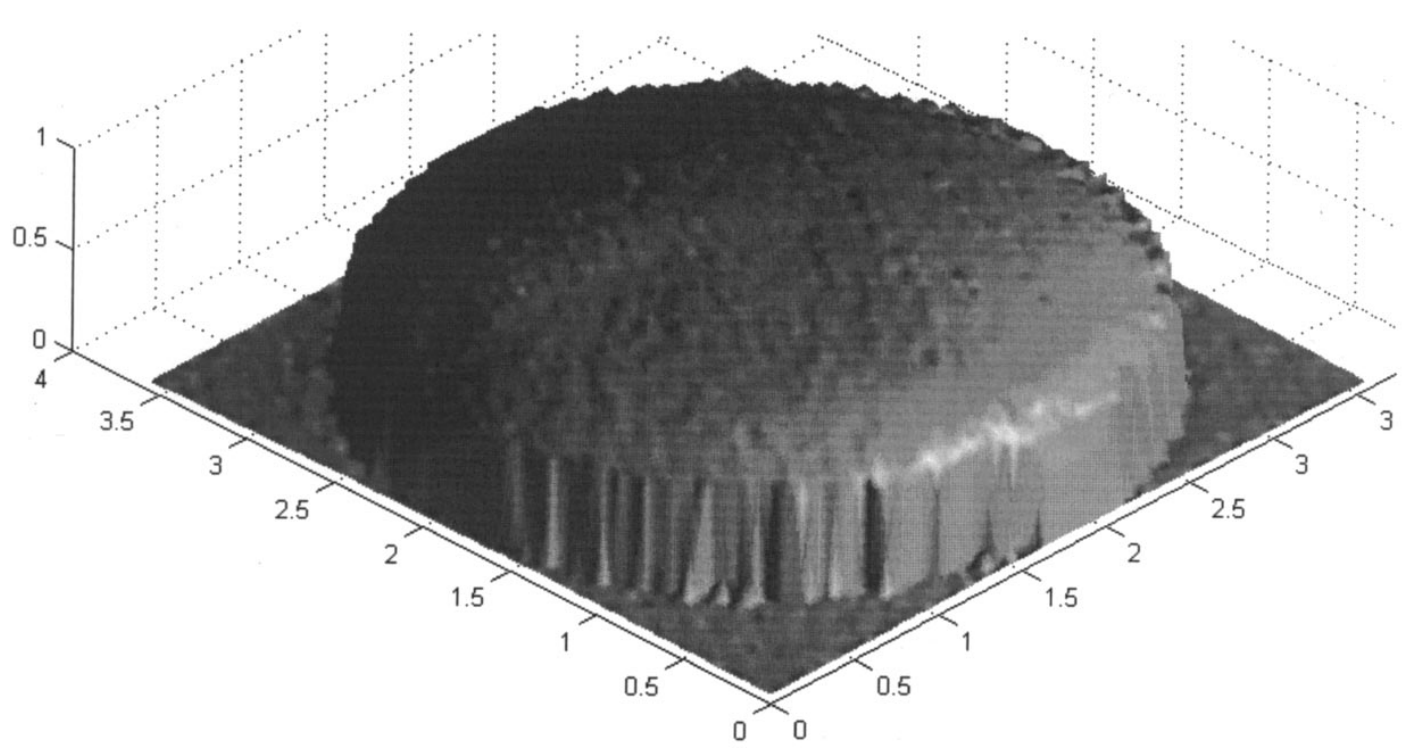

(a)

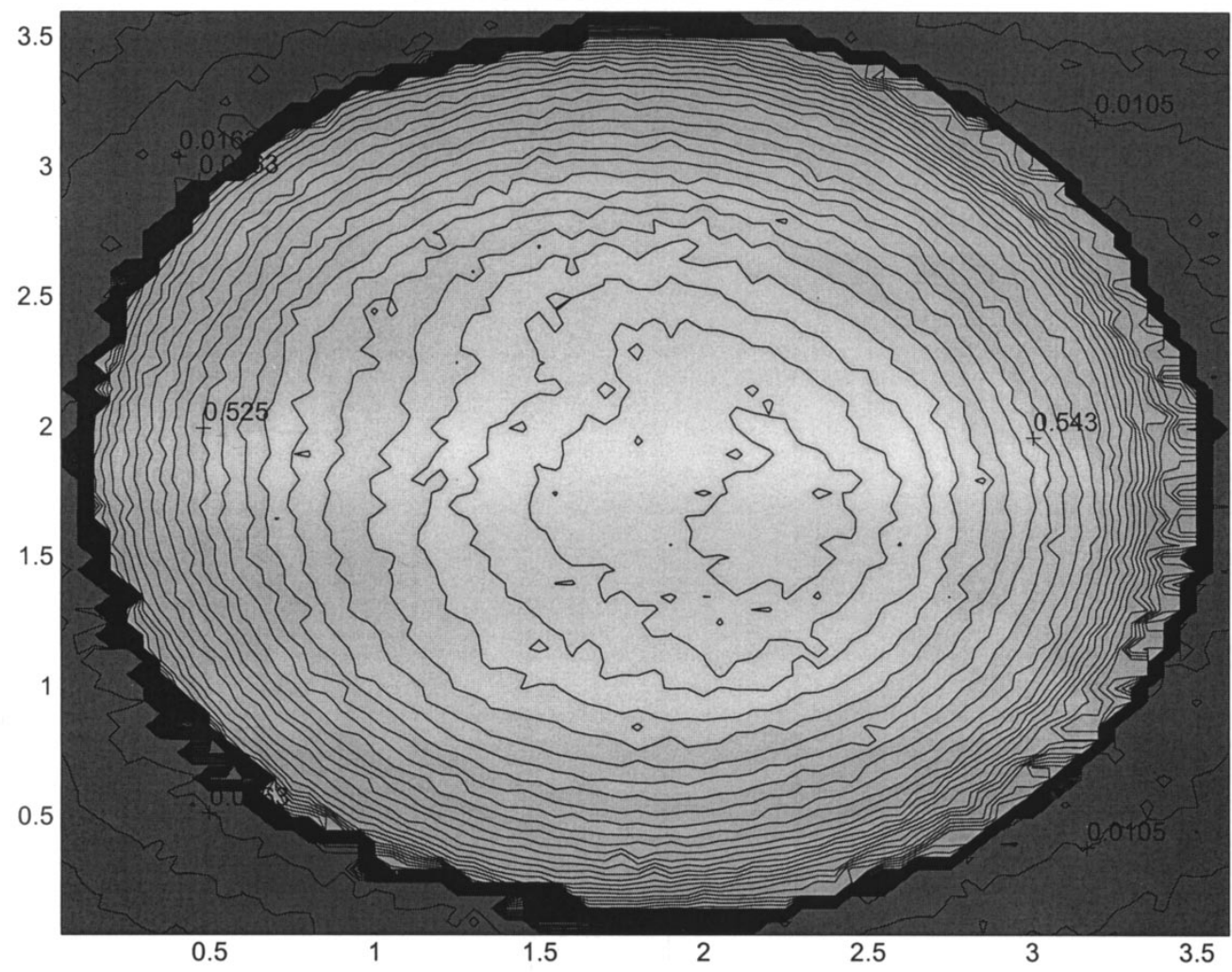

(b)

Fig. 7. (a) New anode. (b) Contour plot of new anode surface.

sphere fitting method used is the four-point sphere fit [5]. In a recent study [6] a number of sphere fitting techniques have been tested and compared on simulated data sets.

\section{Aspheric Surfaces}

There are many surfaces that exhibit nonspherical form, which can include Toric, Parabolic, and Conic surfaces, all of which are possible geometries used with electrical contact surfaces. Form fitting methods have been developed for these geometries, [7].

\section{EXPERIMENTAL METHODS}

To evaluate the laser based measurement system, measurements are made of automotive relay contacts tested in a commercial device. The purpose is to determine the volumetric erosion and to then define a methodology, to aid in the development 


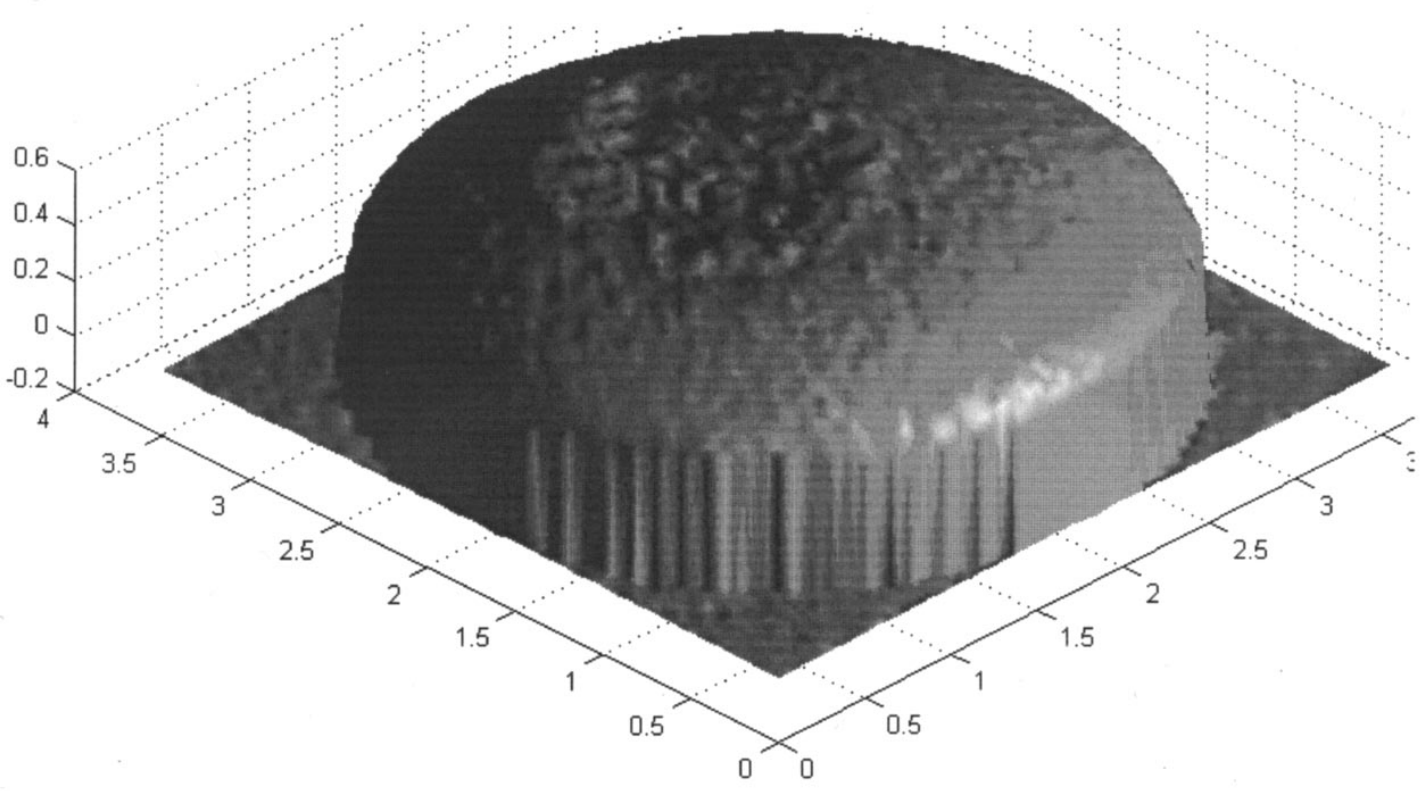

Fig. 8. Contact 1 A. Anode after 500 operations.

of relays for higher dc supply voltages. The results are in two sections.

\section{A. Experiment (1)}

In this initial experiment, comparisons are drawn between the laser system and the results presented in a previous paper, [1], where the main measurement method was stylus based. The contacts tested are of nominal spherical geometry, type B.

\section{B. Experiment (2)}

In this experiment, $\mathrm{AgSnO} 2$ automotive relay contacts have been investigated for erosion profiles. The contacts were tested in a commercial relay to the conditions defined in Table II. The contact material is silver tin indium oxide with $12 \%$ metal oxides. The test cycle is a make and break operation in a $13 \mathrm{~V} \mathrm{DC}$ supply. The load is lamp and inductance (connected in series. The current is $150 \mathrm{~A}$ inrush and $35 \mathrm{~A}$ steady state, [8].

\section{RESULTS}

\section{A. Experiment 1}

Fig. 4 shows an eroded spherical contact of $2.4 \mathrm{~mm}$ radius. The $Z$ axis is magnified to show the curvature of the contact surface.

Fig. 5 shows a data set measured on the stylus system with the $2.4 \mathrm{~mm}$ sphere removed from the raw data. In this case the erosion pattern has been shown previously to correspond to a contact surface where the mass erosion is zero, [1]. The figure is presented here for comparison with Fig. 6, which is a noncontact laser based measurement of the same contact surface. Fig. 6 shows greater surface roughness resulting from the lower $Z$ resolution of the laser system. An evaluation of the volume erosion of the surface above and below the datum surface shows similar results for both systems.

\section{B. Experiment 2}

Results on the erosion of the automotive relay contacts, as defined in Table II. Figs. 7-12 are anode contacts. Fig. 14 is a cathode after 5000 test cycles for direct comparison with Fig. 9, the corresponding anode. Fig. 13 shows the volume calculation of contacts relative to the contact support.

\section{DISCUSSION}

\section{A. Experiment 1}

The results presented here demonstrate the ability of the two systems in the 3-D measurement of electrical contact surfaces. The four-point sphere fit was used to define the $2.4 \mathrm{~mm}$ radius from the measurement of a number of new contacts. A comparison of Figs. 5 and 6, show the effectiveness of both measurement systems. In both cases a $2.4 \mathrm{~mm}$ sphere has been removed from the raw data to leave the level plane surface for $(\Delta V)$ analysis. Fig. 6 is turned $180^{\circ}$ from the data in Fig. 5. The laser measurement in Fig. 6, shows apparently more surface roughness on the plane, this is a result of the lower $Z$ resolution of the probe.

\section{B. Experiment 2}

The laser measurement shown in Fig. 7(a) is for a new contact. A study of new contacts has shown that the welding process used to attach the contact material to the copper base leads to a lack of repeatability in both the initial volume and heights of the contact surface above the copper. A contour map of the surface in Fig. 7(b) shows that the sample tested is inclined upwards to the right of the figure, although the base is flat. This can be shown by the heights shown on the figure, at $0.5 \mathrm{~mm}$ in from the right and left sides, respectively $0.525 \mathrm{~mm}$ and $0.543 \mathrm{~mm}$. Additionally a sphere fit to the surface shows an irregular surface. The edge of contact is irregular as a consequence of the low number of data points.

Figs. 8-11 show anode contact erosion and are based on data arrays of approximately $70 \times 70$. The volumetric erosion 


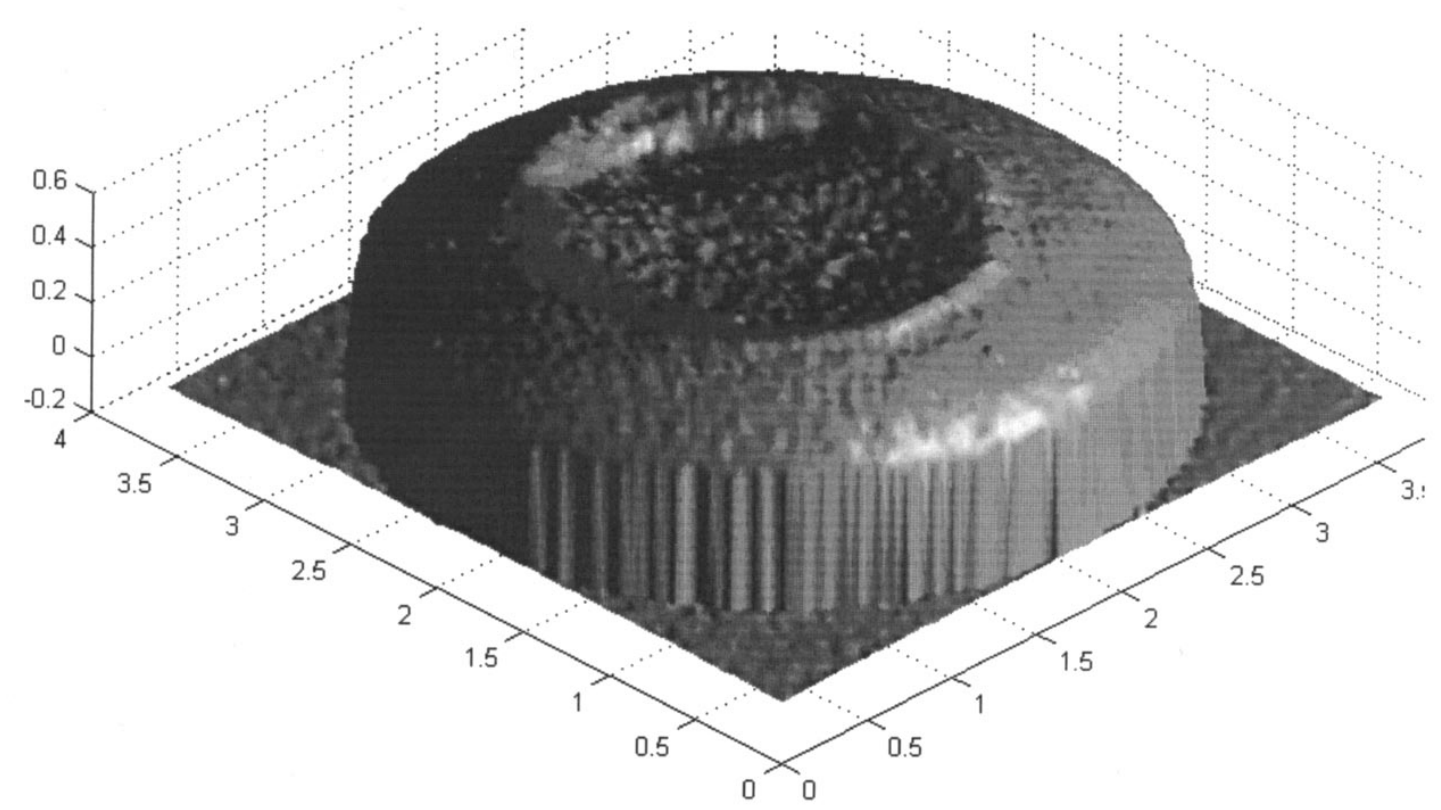

(a)

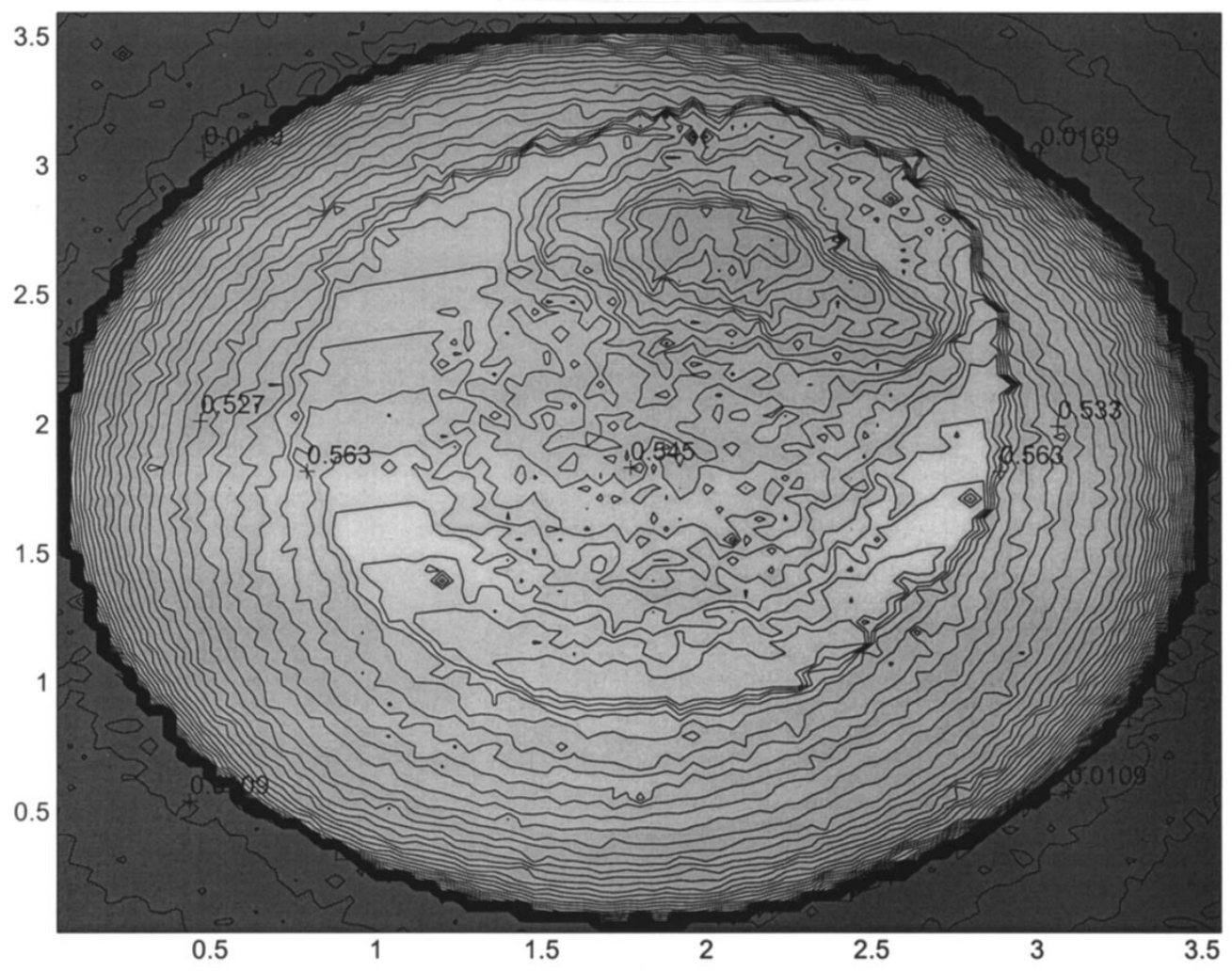

(b)

Fig. 9. (a) Contact 2 A. Anode after 5000 operations. (b) Contour plot of anode after 5000 operations.

is shown in Fig. 14. The surface erosion on Fig. 8, after 500 switching operations ( $1 \mathrm{~A})$ shows a gain in contact volume, over the sample shown in Fig. 7. This is a result of the surface irregularity identified above. The diameter of the erosion area is approximately $1 \mathrm{~mm}$. In Fig. 9(a), after 5000 operations (2 A) the eroded area is shown to be of a larger diameter $(2 \mathrm{~mm})$, but to be of a lower volume when compared to the sample in Fig. 8. Fig. 9(b) is a contour plot of the surface. This shows an interesting feature, in that the expected mass loss of the anode can be observed as the crater toward the top right, within a larger crater of approximately $2 \mathrm{~mm}$ diameter. After 20000 operations (3 A), Fig. 10, the wear area covers most of the 


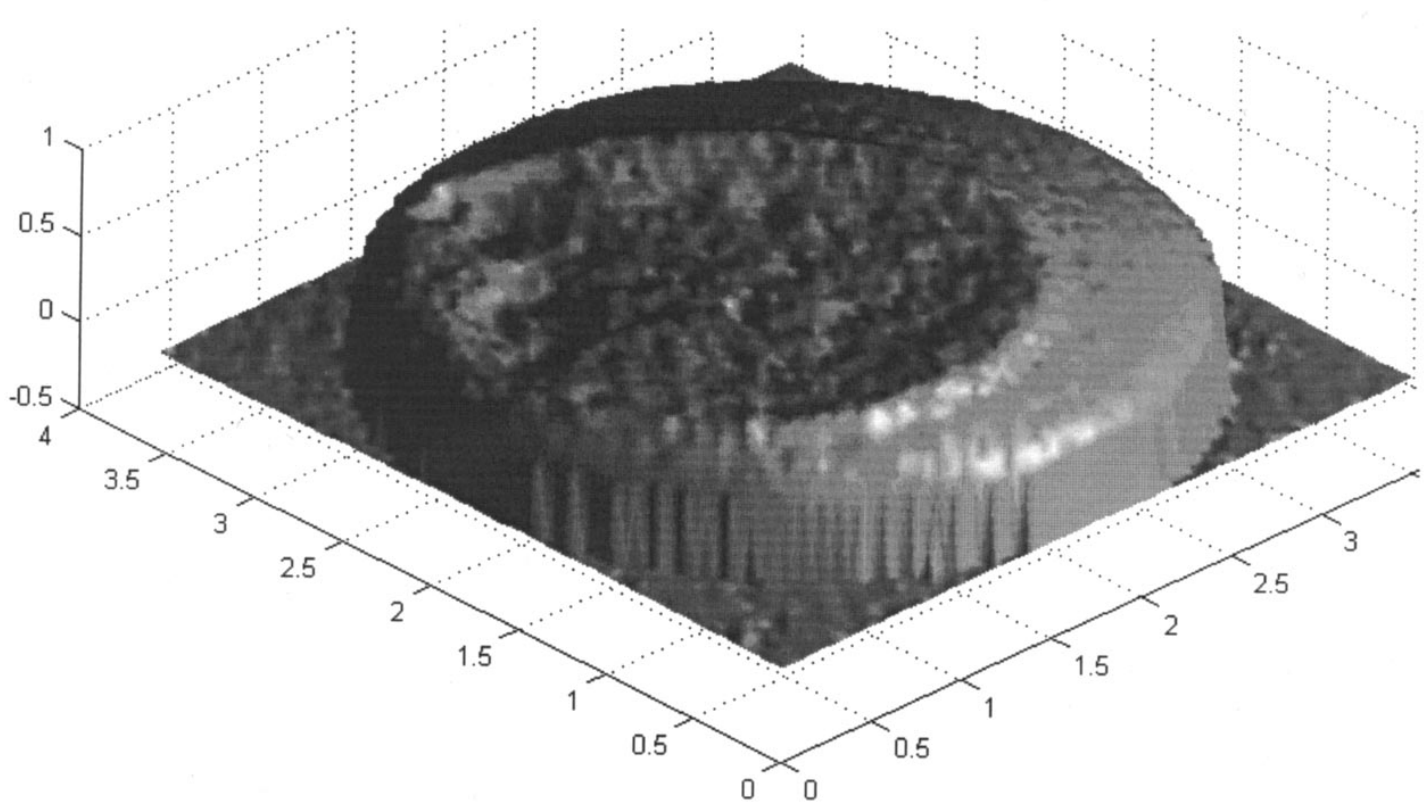

Fig. 10. Contact 3 A. Anode after 20000 operations.

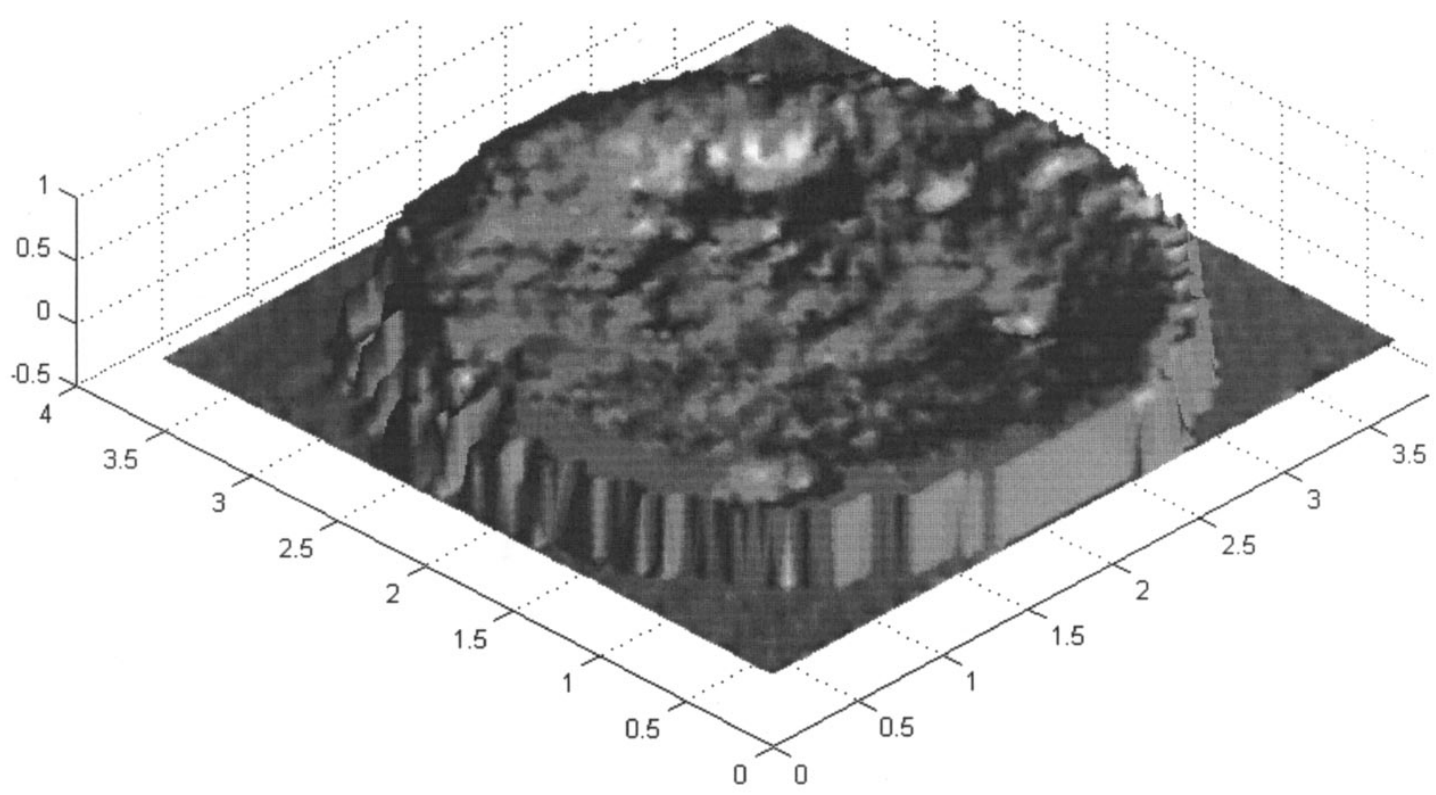

Fig. 11. Contact 4 A. Anode after 300000 operations.

contact surface, and the volume has increased over the new contact sample. After 300000 operations (4 A), Fig. 11, the wear area covers the whole contact surface, and has reduced the height of the contact from the datum surface. This is confirmed in the low value of the volume erosion in Fig. 14.

Fig. 12 shows data collected using the auto-lift-off stylus method and corresponds to Fig. 9. In both cases the higher resolution of the $Z$ axis and the larger data array, typically $120 \times 120$, leads to an improved resolution image. The systematic flanking error associated with the stylus can be observed resulting in the apparent slope on the sides of the contact. The volume calculations are therefore significantly greater. This can be observed in Fig. 13, where for all of the contacts the volume calculation is $0.5 \mathrm{~mm}^{3}$ greater. The additional volume in Fig. 13, due to the flanking error, significantly increasing the volume on contact ( $4 \mathrm{~A}$ ), when compared to the laser measurement. Fig. 14(a) shows the cathode contact after 5000 operations, showing an erosion area comparable with Fig. 9(a) and (b). Fig. 14(b) show a contour plot of the cathode sample. It shows the expected gain on the surface in the form of a pip formation. The main body of the surface although exhibiting an apparent roughening has not been deformed to the same extent as the anode in Fig. 9(b).

\section{Measurement Methodology}

As a result of the discussion of the surface changes the following process is defined for the measurement of irregular 


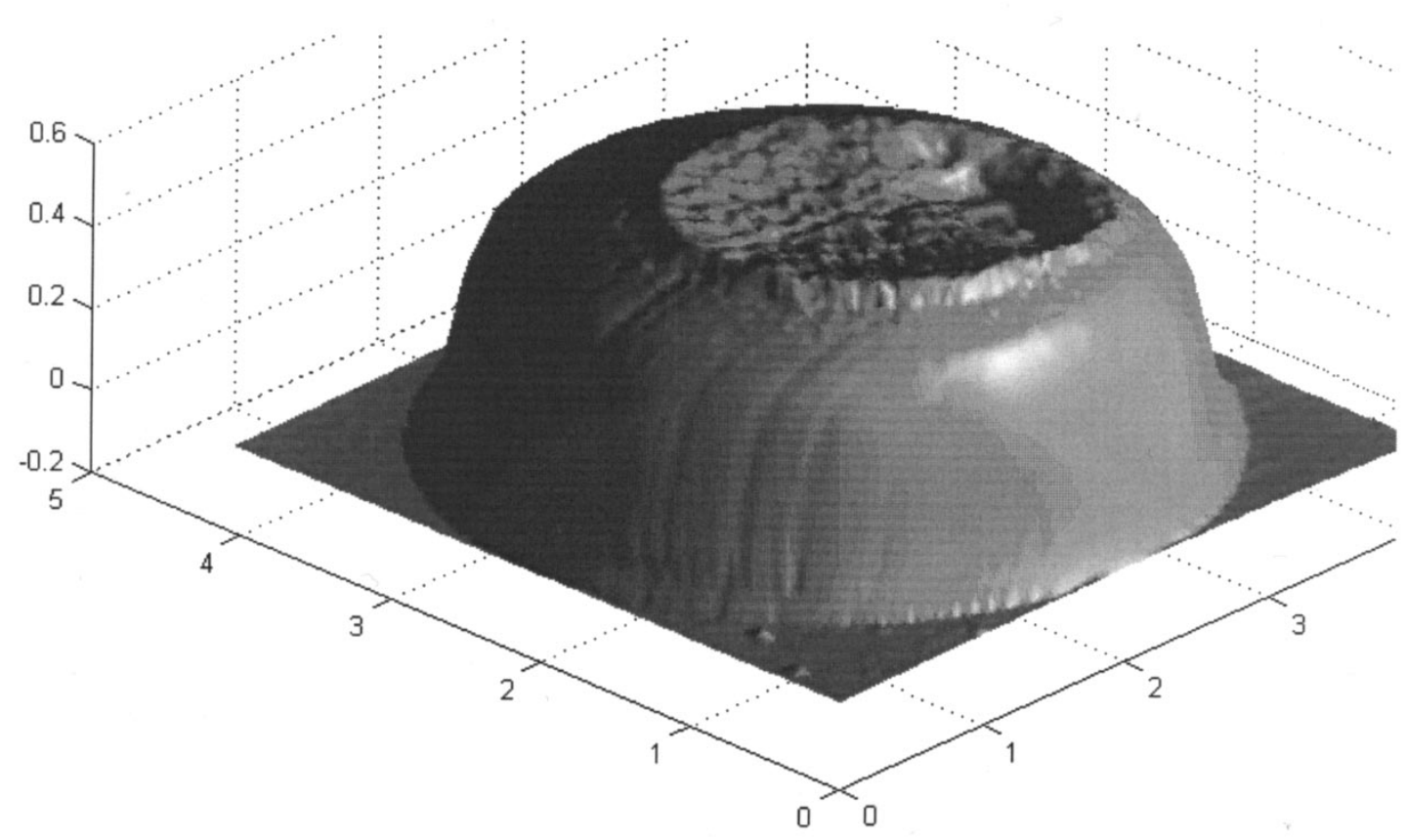

Fig. 12. Contact 2 A. Anode after 5000 operations, using auto-lift off stylus.

contact surfaces, similar to those produced during a welding manufacturing process.

Stage 1. Measure the volume of a new contact, mounted on the copper base.

Stage 2. Assemble the contacts in a commercial device.

Stage 3. Run the required test procedure.

Stage 4. Dismantle the relay device and measure the volume of the contact.

Stage 5. Calculate the difference in volume between stage 1 and stage 4 .

\section{Arc Erosion of Automotive Contacts}

The arc erosion of contacts is of particular interest, when improving the reliability of automotive relays. There have been a number of recent investigations into arc erosion of relay contacts, [8], [9]. This paper does not aim to contribute to the discussion on the performance, but does indicate the complex behavior of the erosion processes in dc automotive relays. The main objective in the development of new improved relay devices is in improving the life of such devices. The relay performance depends on a number of interrelated factors, which are not easily replicated in the laboratory. For example the contact bounce of the device is expected to change as the contacts erode, and the contact bounce characteristics have a relevance to the performance in that one of the main failure modes is welding. It is expected that the theory of contact bounce as given in [10] will not apply to severe surface erosion. In [10] it was shown that a low level of erosion for example Figs. 8 and 9, will not affect the bounce dynamics. This is because the surface properties were proposed to be of more importance than the local geometry. In the case of contacts such as those shown in Fig. 11, where the surface properties will have changed. Then it can be expected that there will be changes in the bounce dynamics and this will be critical in the operation of relay devices with a high in-rush current.

Figs. 9(b) and 13(b) show that the transfer of material is as expected, with the anode loosing mass and the cathode gaining. The arc erosion pattern of the surface indicates that the anode surface is plastically deformed by the impact process during contact bounce. The $2 \mathrm{~mm}$ crater on the anode is a result of the higher temperature of the anode over the cathode. The anode is loosing material because the surface is at a higher temperature, as a result of electron bombardment across the small gap during the bounce. The cathode surface form has not been deformed away from the pip formation. The arcing event can then be seen to be centred around the pip and crater formation, which would be expected to remain in contact for a longer period during the break process since the break will be accompanied by lateral motion of the contact surfaces. During the impact process the arc will be centred at the pip and crater formation and the surface will be heated by conduction.

To improve the long-term performance of relay contacts a detailed study of impact mechanics with surface changes will be required.

\section{CONCLUSION}

This paper presents measurements taken on an auto-focus based high precision measurement system. The results show that the system and the software analysis methods used are able to measure the contact volume changes due to arc erosion.

1) The three measurement systems considered are able to measure the volume changes on contacts with a spherical contact region and with a low level of surface erosion.

2) The laser noncontact system is able to measure the total volume of contacts. This can be used to study the ero- 


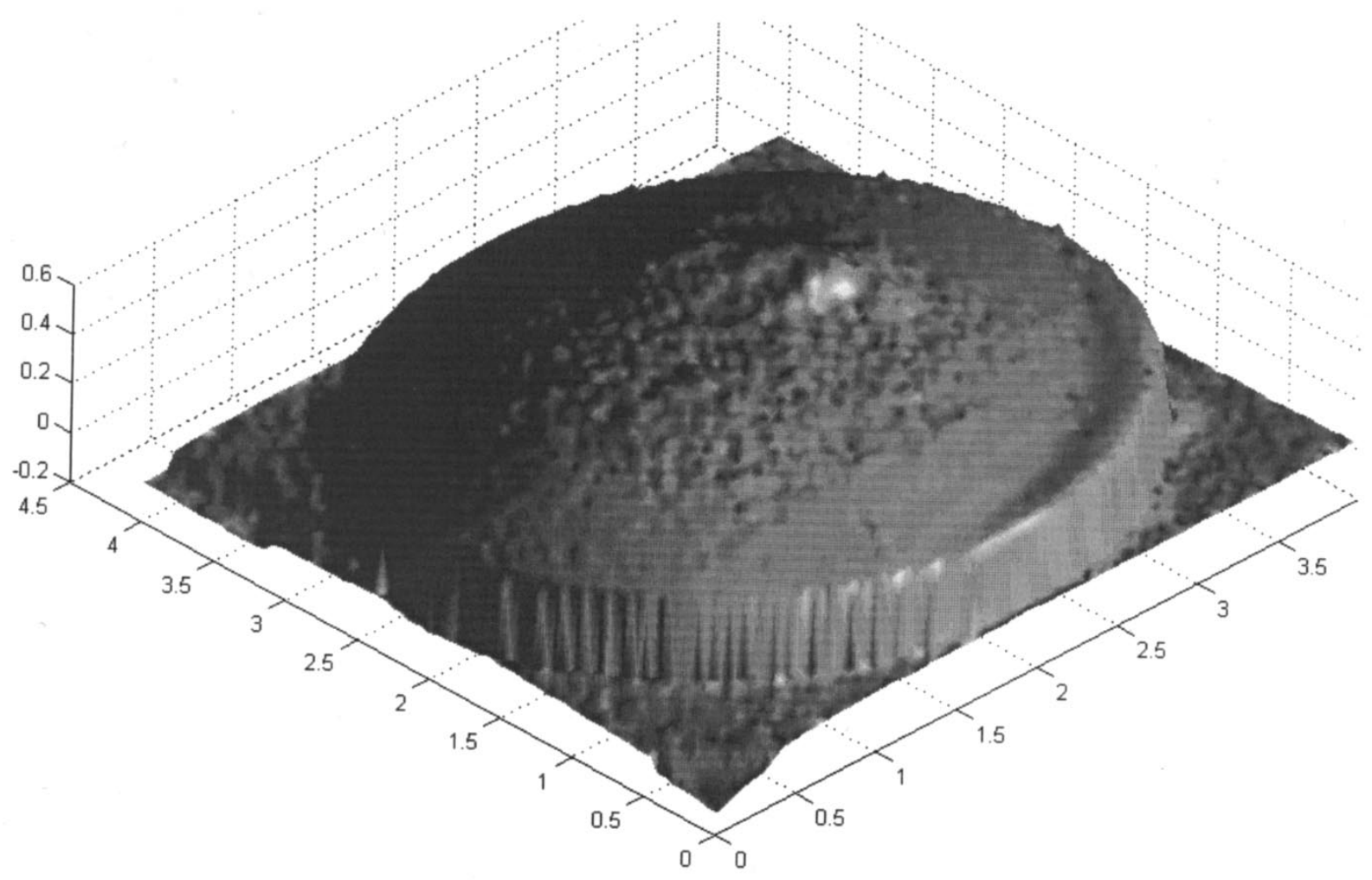

(a)

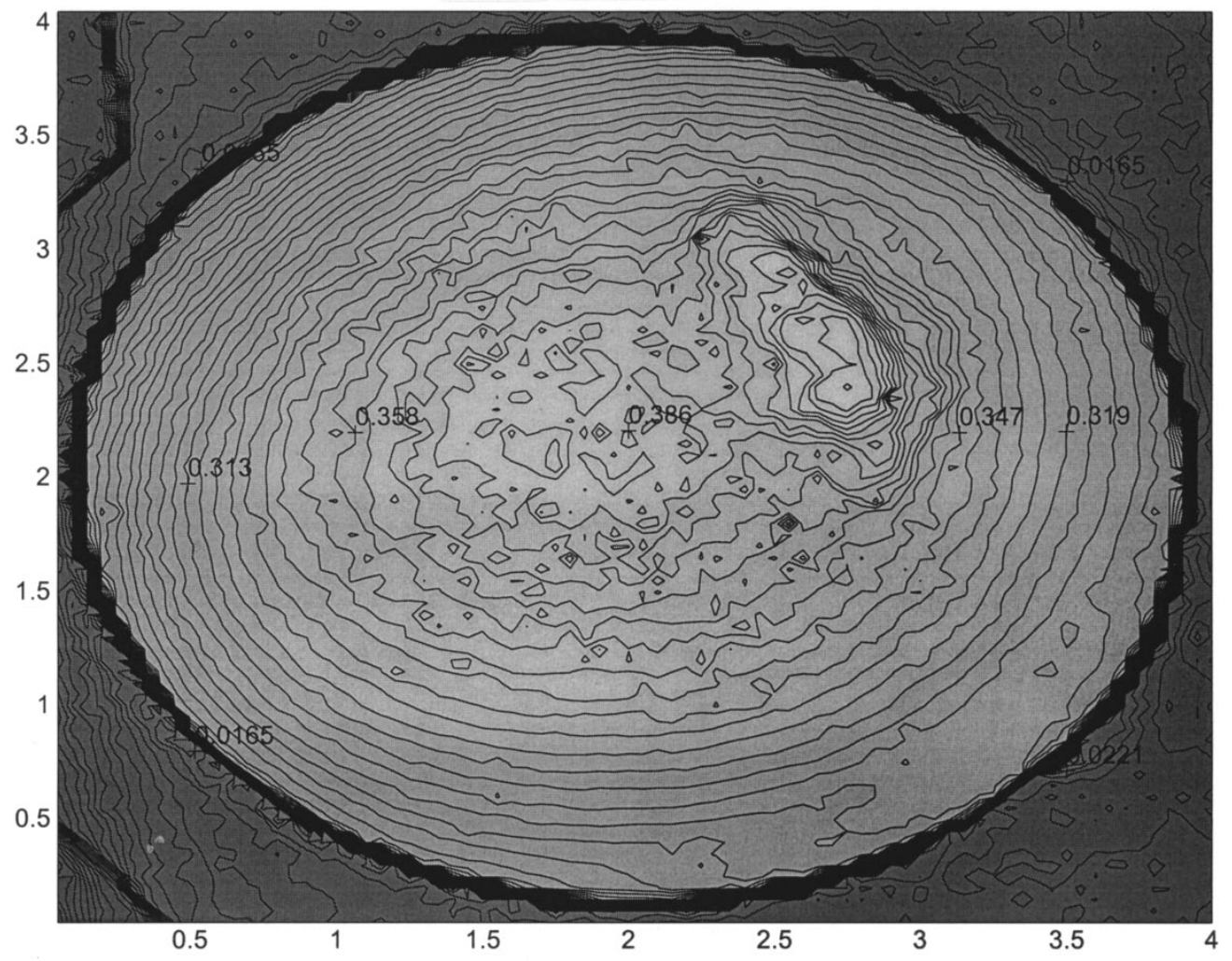

(b)

Fig. 13. (a) Contact $2 \mathrm{C}$, cathode after 5000 operations. (b) Contour plot of $2 \mathrm{C}$, cathode after 5000 operations. 


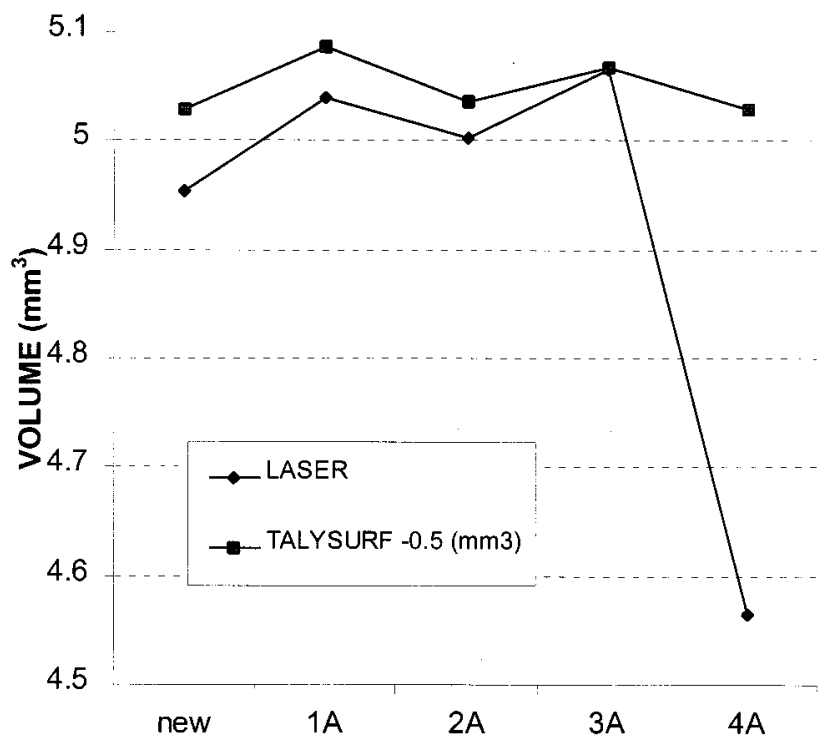

Fig. 14. Volume calculations of anode eroded contacts, based on the 3-D Simpson method, [1].

sion of a range of contact geometries and also severely eroded contacts.

3) To minimize errors in the Volume calculation the data array should be a large as possible.

4) For a full study of contact performance a measurement must be taken relative to the same unused contact, to remove errors associated with irregular contact assembly processes.

5) In the study of automotive relay contacts, the laser system has shown that there is a complex interaction of the anode and cathode surfaces, and that the cathode surface demonstrates a higher temperature profile than the cathode, leading to plastic deformation of the anode surface.

6) The contact erosion will affect the contact bounce dynamics, and thus the erosion trend. Further studies are proposed on severely eroded contacts.

\section{ACKNOWLEDGMENT}

The author would like to thank C. Maul for assistance in developing the measurement system, Dr. S. Chen, Chugai, for useful discussions, and F. McCullough for data collection.

\section{REFERENCES}

[1] J. W. McBride, K. J. Cross, and S. M. Sharkh, "The evaluation of arc erosion on electrical contacts using 3-D surface profiles," IEEE Trans. Comp., Packag., Manufact. Technol., vol. 19, pp. 87-97, Mar. 1996.

[2] E. Hertzmannseder and V. Eckroth K, "Field versus erosion model investigation for contactor contacts," in Proc. ICEC'98, Munich, Germany, Sept. 1998, pp. 349-354.

[3] J. W. McBride, M. Hill, and M. Jungr, "The analysis of the form of aspheric lens surfaces," Proc. Lamdamap'97, pp. 381-390, 1997.

[4] M. Jung, M. Hill, and J. W. McBride, "Surface reconstruction from discrete data obtained by a 3D surface measurement system," Proc. Lamdamap'99, pp. 277-286.

[5] J. W. McBride and K. J. Cross, "The measurement and analysis of the form of curved surfaces," Int. J. Mach. Tool Manufact., pp. 597-610, May 1996.

[6] M. Jung, K. J. Cross, J. W. McBride, and M. Hill, "A method for the selection of algorithms for form characterization of nominally spherical surfaces," Prec. Eng., 2000.

[7] M. Jung, "Precision characterization of 3-D form," Ph.D. thesis, Univ. Southampton, Southampton, U.K., Nov. 1999.

[8] Z. Chen and G. Witter, "Electrical erosion comparison of material for automotive loads," Proc. ICEC'98, Sept. 1998.

[9] N. Ben Jemaa, L. Morin, D. Jeannot, and L. Nedelec, "A study of new contacts materials under break arc in automobile area," Proc. ICEC'98, pp. 89-96, Sept. 1998.

[10] J. W. McBride, "Electrical contact phenomena during impact," Trans. Comp., Hybrids, Manufact. Technol., vol. 15, pp. 184-192, Apr. 1992.

John W. McBride received the M.S. degree in aeronautical engineering from the University of Southampton, Southampton, U.K., in 1978 and the Ph.D. degree in electrical contact phenomena from Plymouth University, Plymouth, U.K., in 1986.

From 1985 to 1987, he lectured in the Mechanical Engineering Department, Plymouth University and from 1987 he has been a Lecturer, Senior Lecturer, and since 1999, Reader in instrumentation and measurement in the School of Engineering Science and the Electrical Engineering Department, University of Southampton. His main research interests include electrical contacts, metrology, and instrumentation.

Dr. McBride is a Chartered Engineer and Chair of the Electro-Mechanical Research Group. 